\title{
La langue bretonne dans la Mission de folklore musical de Basse-Bretagne de 1939
}

The Breton language in the survey conducted by the Mission de Folklore Musical in Lower Brittany in 1939

\section{Gilles Goyat}

\section{OpenEdition}

\section{Journals}

Édition électronique

URL : https://journals.openedition.org/lbl/1832

DOI : $10.4000 / \mathrm{lbl} .1832$

ISSN : 2727-9383

\section{Éditeur}

Université de Bretagne Occidentale - UBO

\section{Édition imprimée}

Date de publication : 1 novembre 2011

Pagination : 87-96

ISBN : 978-2-901737-92-6

ISSN : 1270-2412

\section{Référence électronique}

Gilles Goyat, «La langue bretonne dans la Mission de folklore musical de Basse-Bretagne de 1939 »

La Bretagne Linguistique [En ligne], 16 | 2011, mis en ligne le 01 mai 2021, consulté le 22 mai 2021.

URL : http://journals.openedition.org//bl/1832 ; DOl : https://doi.org/10.4000//bl.1832

\section{cc) (1)}

La Bretagne Linguistique est mise à disposition selon les termes de la Licence Creative Commons Attribution 4.0 International. 
Gilles GOYAT*

\title{
La langue bretonne dans la Mission de folklore musical de Basse-Bretagne de 1939
}

\begin{abstract}
A
u cours des années 1930, les arts traditionnels semblent susciter l'intérêt de certaines institutions nationales qui ressentent la nécessité d'organiser «une recherche musicale ethnologique» basée sur des données recueillies directement sur le terrain. Suite à l'arrivée au pouvoir du Front populaire, le Musée national des Arts et Traditions populaires est créé en 1937. Son premier directeur, l'ethnologue George Henri Rivière, passionné de musique, organise le premier Congrès international de folklore l'année même de la création du musée, et peu après, prend la décision d'envoyer une Mission de folklore musical en Basse-Bretagne.
\end{abstract}

\section{La préparation}

Pourquoi avoir choisi la Basse-Bretagne?

En 1939, la population française reste majoritairement rurale; néanmoins, plusieurs facteurs montrent l'urgence de collecter certains éléments de sa culture traditionnelle. G. H. Rivière est aussi un «amoureux» de la Bretagne, dont le peintre Henri Rivière (1864-

* Doctorant, CRBC Rennes 2/ueb 
1951), son oncle, lui avait fait découvrir toute la beauté, et il semble bien que ce soit lui qui ait choisi de commencer les enquêtes par la Basse-Bretagne. Celle-ci rassemble, en effet, plusieurs caractéristiques qui en font un terrain d'expérimentation quasi idéal: «Personnalité ethnique originale; limites géographiques définies (la mer sur trois côtés; une frontière linguistique pour le quatrième); isolement relatif au cours de l'histoire favorisant la conservation d'états anciens; tradition populaire encore vivante ou présente aux mémoires; riche folklore musical ${ }^{1}$.»

L'équipe de la Mission comprendra une musicologue, Claudie Marcel-Dubois, qui travaille au musée, et aussi un linguiste : il faut que «l'indispensable collaborateur linguiste soit du pays et soit doté de toute la formation scientifique nécessaire, une formation consacrée par les diplômes dans sa spécialité ${ }^{2} »$. G. H. Rivière s'adresse alors aux professeurs de celtique de Paris, $M$. Vendryès et $\mathrm{M}^{\mathrm{me}}$ Sjoestedt Jonval, qui lui recommandent François Falc'hun, le seul étudiant de l'École Pratique des Hautes Études préparant le diplôme de 1'Institut de phonétique qui soit bretonnant de langue maternelle. Ce linguiste doit être capable de noter en phonétique les textes chantés dans les différents parlers; c'est pourquoi G. H. Rivière lui conseille de rendre préalablement visite à Pierre Le Roux, l'auteur de l'Atlas linguistique de la Basse-Bretagne.

Pour préparer cette Mission, «un questionnaire dont le préambule expliquait le but et les méthodes de la mission projetée, fut expédié dans toutes les communes de Basse-Bretagne, aux prêtres, aux instituteurs et aux personnalités connues pour s'intéresser au folklore ${ }^{3} »$. Mais sur un millier de questionnaires expédiés, seulement 106 furent renvoyés. Après dépouillement des réponses, un fichier des informateurs par commune fut dressé, et l'itinéraire de la Mission, tracé dans ses grandes lignes.

Le but premier de la Mission est la prospection de la musique et de la danse populaires, et non la langue en elle-même. La Mission

1. Jean-Michel Guilcher, La tradition populaire de danse en Basse-Bretagne, Paris, école pratique des hautes études, 1963 (première édition), p. 8.

2. George Henri Rivière, lettre du 21 juin 1939 à Xavier de Langlais, archive MBB 39 B2 D3.

3. François FalC'HUN, «Une Mission de folklore musical en Basse-Bretagne», Conférences universitaires de Bretagne, Paris, Les Belles-Lettres, 1943, p. 122. 
utilisera les techniques les plus modernes disponibles à l'époque : enregistreur de disques, appareil photographique et caméra.

Parmi ceux qui n'avaient pas répondu au questionnaire figurent plusieurs membres du mouvement culturel breton, qui se méfient de cette initiative prise à Paris et prônent le boycottage. C'est le cas, par exemple, de l'abbé Jean-Marie Perrot, recteur de Scrignac et dirigeant du Bleun-Brug, le mouvement culturel catholique bretonnant, qui explique son point de vue dans la réponse qu'il adresse le 30 juin, en breton, directement à François Falc'hun : «Ma n'em eus ket respontet kentoc' 'h eo dre ma 'm eus disfiziañs eus kement tra a ra ar c'houarnamant e Breiz... Daou-ugent vloaz a zo ma c'houlennomp diganti ar brezoneg er skol, ha daou-ugent vloaz a zo e vezomp kaset da gaoc'hkezeka.» (Si je n'ai pas répondu plus tôt, c'est parce que je me méfie de tout ce que fait le gouvernement en Bretagne... Cela fait quarante ans que nous lui demandons l'enseignement du breton à l'école, et cela fait quarante ans qu'il nous envoie paître) ${ }^{4}$. Peu après, François Falc'hun a la confirmation qu' «un mot d'ordre venu de Paris, et répandu déjà dans les milieux du Bleun-Brug, recommandait l'abstention, la non-collaboration à notre mission ${ }^{5}$ ».

Finalement, suite aux interventions personnelles de militants convaincus de l'intérêt de la Mission et à la publication de plusieurs communiqués de presse, dont ceux des Semaine(s) religieuse(s) des évêchés de Quimper, Vannes et Saint-Brieuc, ces défenseurs de l'enseignement de la langue bretonne se ravisent et l'abbé Perrot, comme plusieurs autres, finit par aider activement la Mission.

\section{Le déroulement}

Le terrain fut préalablement préparé par des correspondants locaux: des prêtres, comme l'abbé Louis Le Cam, recteur de Brandérion ou l'abbé Jean-Marie Perrot, mentionné ci-dessus, des instituteurs publics comme Jean Delalande(Kerlann) à Saint-GuénoléPenmarc'h, ou les frères Goraguer à Plogastel-Saint-Germain, ou encore des personnalités comme le peintre Xavier de Langlais à Surzur ou Yves Le Goff (Yeun ar Gow), notaire à Gouézec... «Ils

4. Jean-Marie Perrot, lettre du 30 juin 1939 à F. Falc'hun.

5. Archives François Falc'hun, Centre de recherche bretonne et celtique, Brest, FAL1 M75, p. 1. 
usèrent de toute leur influence, parcourant souvent la campagne pour nous recruter des chanteurs $\left.{ }^{6}.\right\rangle$

Du 8 au 15 juillet, avant que ne débute la Mission, François Falc'hun fait, grâce à l'entremise de Loeiz Herrieu, un stage linguistique à Brandérion, afin de se familiariser avec le breton vannetais «qu'[il] lisait sans l'avoir jamais entendu parler ${ }^{7} »$. Un mois plus tôt, il avait reçu de L. Herrieu la réponse suivante : «Je crois avoir trouvé ce qu'il vous faut : mon ami et collaborateur M. l'abbé Le Cam, recteur de Brandérion par Hennebont, (ancien officier de la Grande Guerre, Légion d'honneur, camarade de J. P. Calloc'h, auteur de plusieurs ouvrages sur la région et folkloriste lui-même) veut bien vous offrir l'hospitalité. Vous trouverez chez lui, en plus de l'accueil cordial du meilleur des confrères, toutes les facilités pour vous mettre au courant de la prononciation du vannetais. L'abbé Le Cam est, en effet, de l'Arvor du pays de Vannes. Il vous renseignera donc sur la prononciation de la partie maritime du Pays. Son vicaire, un de mes bons amis et collaborateurs, est du Bas-Vannetais et vous sera précieux pour la prononciation de l'Argoed. En vous aidant de mon Breton Usuel, il vous sera possible de vous assimiler assez (sic) la prononciation du vannetais pour pouvoir accomplir votre mission ${ }^{8}$.» Mais ce court séjour ne fut évidemment pas suffisant pour lui permettre de parvenir à une parfaite compréhension des parlers vannetais.

Le 15 juillet, François Falc'hun se rend à Surzur où il retrouve les deux autres membres de la Mission (Claudie Marcel-Dubois et Jeannine Auboyer, technicienne et preneuse de vues, aussi chargée de la tenue du journal de route); celles-ci se déplacent dans une petite automobile transportant aussi le matériel d'enregistrement, y compris les accumulateurs indispensables dans les maisons pas encore reliées au réseau d'électricité, le tout pesant environ $200 \mathrm{~kg}$ ! François Falc'hun les suit à bicyclette; quand la distance est importante et que c'est possible, il charge son vélo dans le train ou dans le car.

La Mission parcourt le sud de la Basse-Bretagne de Surzur à Plozévet, avec des détours dans l'Argoat (voir : Itinéraire et calen-

6. François FALC'HUN, «Une Mission de folklore musical en Basse-Bretagne», op. cit., p. 126.

7. François FALC'HUn, ibid., p. 124.

8. Loeiz Herrieu, lettre du 29 mai 1939 à F. Falc'hun. 
drier de la Mission, ci-dessous); mais plusieurs localités prévues n'ont pas été visitées, faute de temps. Partout l'accueil est cordial; contrairement à Pierre Le Roux et à Loeiz Herrieu lors de leurs enquêtes linguistiques quelques années auparavant, ils n'essuient pas de rebuffades.

Une partie du succès de la Mission est due aux enregistrements sonores qu'ils réalisent : la possibilité de réentendre sa voix en a encouragé plus d'un et plus d'une à chanter devant le microphone. Collectage approfondi en pays vannetais, principalement à Surzur et Brandérion, sondages plus superficiels en Cornouaille.

La mobilisation générale ayant été décrétée, la Mission est interrompue le 26 août, sans avoir pu mener son programme à terme. L'équipe est rappelée à Paris.

\section{La collecte}

198 chansons de longueur variable ont été recueillies, de 118 informateurs différents, et enregistrées sur 93 disques de $25 \mathrm{~cm}$ de diamètre. Parfois, plusieurs chansons très courtes ont été gravées sur une même face de disque. De plus, 437 clichés ont été pris, et 12 films muets de quelques minutes chacun, tournés, montrant des danses traditionnelles des terroirs visités.

Sur les quelque 200 chansons (seules les chansons de tradition orale étaient recherchées), quelques-unes semblent inédites, mais la plupart sont déjà connues grâce aux collections publiées, surtout celles de Luzel, et celles de Loeiz Herrieu pour le Vannetais.

Pendant l'enregistrement, F. Falc'hun transcrit les paroles en utilisant les signes phonétiques de l'alphabet dit des romanistes (encore appelé «notation Rousselot»), qui est également celui employé par P. Le Roux dans l'Atlas linguistique de la Basse-Bretagne. Mais il lui arrive parfois d'employer aussi quelques symboles de l'alphabet dit des comparatistes (Bœhmer). C'est évidemment à C. Marcel-Dubois qu'il revient de noter les mélodies.

Bien que le but de la Mission soit de collecter le répertoire des chansons, tant les paroles que les mélodies, elle renseigne aussi F. Falc'hun sur les caractéristiques des différents parlers rencontrés. Il faut noter à ce sujet que, pour la première fois, une même mission de collectage s'intéresse à la fois au Vannetais et aux pays de 
Cornouaille, Léon et Trégor d'une part, et que paroles et mélodies sont notées, et aussi enregistrées, simultanément, d'autre part, ce qui constitue un progrès certain par rapport aux pratiques antérieures : «Pour ne parler que du point de vue linguistique, tout le monde sait combien nos signes phonétiques, si précis soient-ils, sont loin de reproduire les mille nuances de la langue parlée, ces intonations, cette nonchalance ou cette vivacité, ce timbre de voix, cette fermeté ou cette mollesse des articulations qui indiquent à elles seules le lieu d'origine, l'âge, le sexe, et jusqu'au tempérament du sujet parlant. Les notations phonétiques ne nous montrent qu'un squelette.

De plus, il est des phonèmes, des sons, qu'on est bien embarrassé pour noter. Sur mes cahiers, je vois tel mot prononcé trois fois, dont une consonne a été transcrite la première fois par [1], la deuxième par [d], et la troisième par [r]. Le chanteur n'avait sans doute prononcé aucun de ces trois phonèmes, mais quelque chose qui tient des trois à la fois. Et ce n'est là qu'un exemple entre cent. Ce sera une aubaine pour un linguiste que d'entendre à volonté ces phonèmes intermédiaires, que présentent peu de langues, et qui expliquent bien des évolutions au premier abord déconcertantes, comme le passage direct de [d] à [r] aux environs de Surzur, ou de [r] à [d] à Pleyben ${ }^{9}$.»

Mais parce que le niveau de la langue utilisée dans les chansons est plus élevé que celui du parler courant, et pour «d'autres causes d'altérations propres à la langue chantée en tant que telle, les textes de chansons ne pourront être utilisés qu'avec discernement dans les études de dialectologie ${ }^{10} »$. F. Falc'hun fait aussi remarquer que : «Les chansons voyagent beaucoup. Chaque chanteur les chante dans son parler, mais il y a des particularités dialectales qui sont conservées par la rime ou des nécessités métriques. Ces mots dialectaux ne sont pas toujours compris, sont parfois déformés et rendus méconnaissables ${ }^{11}$.»

La Mission a aussi montré qu' «il a existé autrefois un répertoire de chansons commun à toute la Basse-Bretagne. [...] Quantité de

9. Archives François Falc'hun, Centre de recherche bretonne et celtique, Brest, FAL2 M92, p. 5-6.

10. François FALC'HUN, «Une Mission de folklore musical en Basse-Bretagne», op. cit., p. 129-130.

11. Archives François Falc'hun, Centre de recherche bretonne et celtique, Brest, FAL2 M92, p. 6. 
thèmes recueillis par Luzel dans le Tréguier l'ont été par nous en Cornouaille ou au pays de Vannes; quelques-uns figuraient déjà au Barzaz-Breiz. [...] Les difficultés du dialecte vannetais, qui arrêtèrent tant d'enquêteurs KLT (Cornouaille-Léon-Trégor), ne constituaient donc pas une gêne pour la migration des chansons, ni peut-être des chanteurs eux-mêmes autrefois ${ }^{12}$ ».

Une autre conclusion importante est que : «La frontière linguistique elle-même ne joue pas le rôle de cloison étanche, et ce ne sera pas le moindre résultat de la mission de 1939 que de poser nettement le problème des relations du folklore oral de Basse-Bretagne avec celui de la Haute-Bretagne ${ }^{13}$.»

\section{Les suites de la Mission}

Le premier rapport scientifique sur la Mission, comprenant trois parties, la musique, les images et le breton, est rédigé en décembre 1939: "La mission rapporte plus de quatre cents pages qui représentent un intérêt linguistique de tout premier ordre. Pour la première fois, on possède des notations phonétiques d'une certaine étendue concernant le parler de vingt-cinq localités bretonnantes. C'est un apport inappréciable pour la dialectologie bretonne, et pour l'étude des particularités de la langue chantée par rapport à la langue parlée ${ }^{14} . »$

Un plan de travail prévoyant le traitement des données du terrain est mis au point en septembre 1941. Des copies des disques sont réalisées, et une fiche de synthèse est établie pour chaque chant.

F. Falc'hun vérifie, grâce aux disques, les transcriptions phonétiques faites «à la volée» et les recopie. Il réalise les traductions françaises des chansons. Les disques et les photos sont inventoriés, mais pas les films, et la danse est, de ce fait, délaissée.

12. François FALC'HUN, «Une Mission de folklore musical en Basse-Bretagne», op. cit., p. 127.

13. François FalC'HUn, ibid., p. 128.

14. François FALC'HUN, «Rapport sur une mission sur le folklore musical en BasseBretagne», 12 décembre 1939, Les archives de la Mission de folklore musical en Basse-Bretagne, Paris, CTHS et Rennes, Dastum, 2009, p. 365. 


\section{Articles et conférences}

Dès septembre 1940, des conférences sont données et des articles, écrits sur la Mission. Nous ne mentionnerons que ceux qui traitent de la langue. En mars 1942, F. Falc'hun rédige un article sur "Le tailleur berné», qu'il considère comme le meilleur chant rapporté par la Mission. En novembre de la même année a lieu une remise solennelle des copies de l'ensemble des photos et des disques de la Mission à la Faculté des Lettres de Rennes; à cette occasion, F. Falc'hun prononce une conférence sur la Mission, qui sera publiée l'année suivante dans les Conférences universitaires de Bretagne, dont sont extraites plusieurs citations de cet article.

En octobre 1943, C. Marcel-Dubois et F. Falc'hun font ensemble une conférence intitulée «Une chanson de Basse-Bretagne» à l'École du Louvre, à Paris.

En juillet 1949 et en juillet 1954, C. Marcel-Dubois donne des conférences sur «La chanson en Basse-Bretagne» à Quimper, dans la cadre des Fêtes de Cornouaille.

\section{Tentatives de publication et enfin, publication tardive}

En 1941, C. Marcel-Dubois songe à faire des matériaux musicaux recueillis le sujet d'une thèse de musicologie, mais y renonce finalement.

En 1943, l'édition d'un volume de 985 pages portant le titre «Matériaux d'une Mission de folklore musical» n'aboutit pas parce que les documents, tant linguistiques que musicaux, ne sont pas prêts.

La publication est ensuite différée en raison de la guerre, de ses suites, puis de la préparation des thèses de doctorat de F. Falc'hun, $\mathrm{du}$ refus de sa thèse principale en septembre 1944, et aussi de ses graves problèmes de santé, dont l'ablation d'un poumon en 1947 est une des conséquences. Il doit ensuite préparer une nouvelle thèse principale, ce qui lui prend plusieurs années de travail. G. H. Rivière fait alors appel, avec l'accord de F. Falc'hun, à l'un des étudiants de celui-ci, Pierre Denis, pour aider à la copie au propre des textes bretons, en vue de leur publication. En fait, seules les chansons vannetaises avaient été recopiées au propre, en notation phonétique et en orthographe traditionnelle, et étaient prêtes pour l'édition. Plus 
tard, trois nouvelles tentatives de publication $(1962,1972$ et 2001) échouent pour des raisons diverses.

Les documents, sonores, visuels et écrits, n'ont finalement été publiés qu'en novembre 2009, sous forme numérique, sur le DVD-Rom joint au volume Les archives de la Mission de folklore musical en Basse-Bretagne de 1939, à l'occasion du 70 anniversaire de la Mission et du $100^{\mathrm{e}}$ anniversaire de la naissance de François Falc'hun.

ITINÉRAIRE ET CALENDRIER DE LA MISSION DE FOLKLORE MUSICAL en Basse-Bretagne DE 1939

- Juillet (Morbihan)

15 : arrivée à Surzur

16-19, 21 : Surzur et environs

20, 24 : La Tour-du-Parc

22 : Sarzeau et presqu'île de Rhuys

23 : Theix

27-28, 30-31 : Brandérion

- Août (Finistère, sauf les lieux marqués (56), qui sont dans le Morbihan)

$1^{\text {er }}$ : voyage de Brandérion (56) à Penmarc'h

3-4: Penmarc'h

6: Plozévet

7 : Plogastel-Saint-Germain

8 : Plonéis

10 : Quimper (hospice)

11 : voyage à Gourin (56)

12 : Carhaix (hospice) et Le Saint (56)

13 : journée occupée par la moisson

14 : Le Faouët (56)

15 : pardon de Quelven (en Guern, 56)

16 : Saint-Goazec

17 : Châteauneuf-du-Faou 
18, 20 : Gouézec

19 : Pleyben (pas de collectage)

21 : les membres de la Mission assistent à un mariage à Plomodiern

22 : Hanvec, Le Faou

23 : Scrignac

24 : Le Cloître-Pleyben

25 : La Feuillée

26 : voyage de Châteauneuf-du-Faou à Pont-Aven. Fin de la Mission.

- Communes qui devaient être prospectées, mais ne l'ont finalement pas été :

Côtes-du-Nord : Belle-Isle-en-Terre et environs, Saint-Nicolas-duPélem et environs.

Finistère : Plougasnou et environs, Lanildut et environs, Ouessant et Molène, Scaër et environs.

Morbihan : Carnac, Auray et Sainte-Anne d'Auray, Pontivy et environs.

\section{Bibliographie}

FALC'HUN François, «Une Mission de Folklore musical en BasseBretagne», 1943, Conférences universitaires de Bretagne, Paris, Les Belles Lettres, 1943, p. 115-137.

GUILCHER Jean-Michel, La tradition populaire de danse en BasseBretagne, Paris, École pratique des hautes études, 1963 (première édition).

LE GONIDEC Marie-Barbara (dir.), Les archives de la Mission de folklore musical en Basse-Bretagne de 1939, Paris, éditions du CTHS et Rennes, Dastum, 2009.

LE DÛ Jean, «François Falc'hun, 1909-1991», dans BRUN-TRIGAUD Guylaine, LE BERRE Yves et LE DÛ Jean, Lectures de l'Atlas linguistique de la France de Gilliéron et Edmont, Du temps dans l'espace, Paris, éditions du CTHS, 2005, p. 11-17. 